\title{
A preliminary analysis of the suppressive effects of denatonium saccharide
}

\author{
STEPHEN F. DAVIS, LISA A. CUNNINGHAM, TOM J. BURKE, and M. MELISSA RICHARD \\ Emporia State University, Emporia, Kansas
}

and

\author{
WILLIAM M. LANGLEY and JOHN THEIS \\ Butler County Community College, El Dorado, Kansas
}

\begin{abstract}
Two experiments were conducted to evaluate the aversive effects of denatonium saccharide. In Experiment 1, four concentrations of denatonium saccharide were employed in a single-bottle testing procedure that allowed rat subjects access to fluids once daily. Transient suppression effects were shown only by the most potent concentrations. In Experiment 2, the addition of an alternate source of water administered $2 \mathrm{~h}$ after experimental testing resulted in strong, lasting suppressive effects under single- and two-bottle testing procedures. The results of these experiments suggest that denatonium saccharide has excellent potential as a deterrent.
\end{abstract}

Denatonium saccharide, a newly discovered chemical, is the most bitter known to man. It retains a bitter taste in a concentration of 1 part per 20 million parts, and is five times more bitter than the related compound denatonium benzoate and 3,000 times more bitter than quinine. In addition to the sheer curiosity generated by such unusually bitter compounds, denatonium benzoate and denatonium saccharide offer several attractive possibilities for "real-life" application. For example, in addition to its current use as a deterrent to thumb-sucking and nailbiting, researchers at the Proctor \& Gamble Company have reported that the addition of denatonium benzoate significantly inhibited consumption of liquid detergent by preschool children (Berning, Griffith, \& Wild, 1982). It is tempting to speculate that the addition of such substances. might curtail the consumption of other harmful substances, such as paint, paint thinner, turpentine, and denatured alcohol. In this regard it is interesting to note that in 1972 a United States patent was awarded to Walter Minkoff of the Peerless Paint Company for a special paint composition containing denatonium benzoate. Also, the presence of such bitter substances in plastics such as the coverings of electrical lines or computer cables, trash cans, and garbage bags could prevent damage and destruction produced by gnawing rodents and scavenging cats and dogs. Certainly, it appears reasonable to propose that the most bitter substance would result in the greatest aversion and suppression of behavior. Therefore, specific

\footnotetext{
Requests for reprints may be addressed to W. M. Langley, Department of Biology, Butler County Community College, El Dorado, KS 67042, or to S. F. Davis, Department of Psychology, Emporia State University, Emporia, KS 66801. Appreciation is extended to Atomergic Chemetals Corporation, 100 Fairchild Ave., Plainview, NY 11803, for supplying the denatonium saccharide.
}

attention was given to denatonium saccharide in the present set of experiments.

At present there exist no systematic studies dealing with the aversive effects of denatonium saccharide in rodents. The present series of experiments was designed to provide preliminary data in this area.

\section{EXPERIMENT 1}

In this experiment we compared the fluid consumption of groups of laboratory rats that received different concentrations of the chemical. The gathering of such information represents an important first step toward isolating specific concentrations that warrant additional testing.

\footnotetext{
Method

Subjects. Sixteen male albino rats purchased from the Holtzman Company, Madison, Wisconsin, served as subjects. The animals were 90 days old at the beginning of the experiment. All animals were individually housed in wire mesh cages in the vivarium. Food was freely available for the duration of the experiment.

Apparatus. All testing took place in the home cage.

Procedure. Six days prior to the start of experimental testing, all animals were placed on water deprivation and were given access to water for $10 \mathrm{~min}$ at approximately the same time each day. Prior to baseline evaluation, the subjects were randomly assigned to one of four groups $(n=4)$. Each group, in turn, was randomly assigned to a specific denatonium saccharide concentration to be administered during testing: Group 1 received 1 part denatonium saccharide per 1,000 parts water, Group 5 received a 1 part per 5,000 solution, Group 10 received a 1 part per 10,000 solution, and Group 100 received a 1 part per 100,000 solution.

The 5 days immediately preceding testing constituted the baseline consumption period. During the ensuing 15-day test period each animal received five exposures to the following 3-day fluid-administration cycle: On the first 2 days, water was available during the 10-min fluidaccess period; on the 3rd day, only the appropriate denatonium saccharide solution was available during this period. In all cases, fluids were presented in graduated $50-\mathrm{ml}$ centrifuge tubes fitted with spill-resistant sippers.
} 


\section{Results}

Group mean fluid consumption scores in milliliters for the last 2 days of baseline and the test phase are shown in Figure 1. A separate split-plot analysis of variance incorporating groups $(1,5,10$, and 100$)$ as the betweensubjects factor and days as the within-subjects factor was performed on the consumption scores. The NewmanKeuls procedure was used to further probe significant main effects and interactions.

There was no significant difference among the groups during the baseline phase $[F(3,12)=.909, p>.05]$. Figure 1 shows that when denatonium saccharide was presented every 3rd day during the test phase, there was often a decrease in the amount of fluid consumed. Statistical analyses of these data showed significant differences for the effects of days $[F(14,168)=9.53, p<.001]$ and groups $\times$ days $[F(42,168)=3.79, p<.001]$. NewmanKeuls tests indicated that Groups 1 and 5 consumed significantly $(p<.01)$ less than Groups 10 and 100 on Day 3 (i.e., the first presentation of denatonium saccharide). Denatonium saccharide suppressed fluid consumption $(p<.01)$ on additional days only in Group 1, and then only for Days 6 and 9. Group 1 also consumed significantly $(p<.05)$ more water on Days 10,11 , and 13 .

\section{Discussion}

These results show that the four concentrations of denatonium saccharide used in this study were not equally effective in suppressing fluid intake. Only the 1 part per 1,000 and 1 part per 5,000 solutions produced any noticeable reduction in fluid consumption.

Furthermore, the suppressive effects of denatonium saccharide appeared to be transient. Fluid consumption was suppressed by denatonium saccharide for the first three presentations in Group 1 and for only the first one in Group 5.

\section{EXPERIMENT 2}

It appears that the presentation of only one bottle for a period of only $10 \mathrm{~min}$ in Experiment 1 was restrictive and may have created an artificial situation. It can be argued that allowing the animals access to only a single fluid

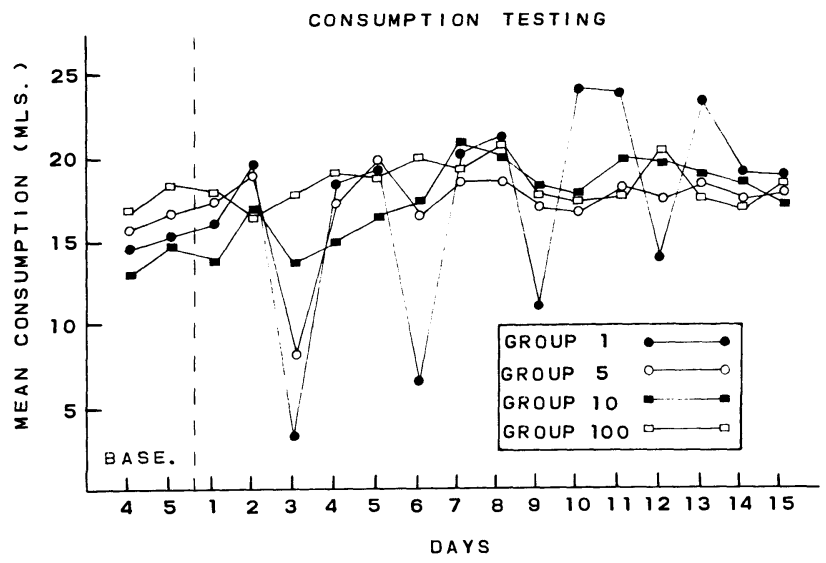

Figure 1. Mean fluid consumption scores (in milliliters) of Groups $1,5,10$, and 100 for the last 2 days of baseline and the test phase, Experiment 1. Denatonium saccharide was presented on Days 3, 6, 9,12 , and 15 during the test phase. once daily may have forced them, due to intensified survival needs during the later stages of training, to consume aversive concentrations of denatonium saccharide that they might not otherwise have consumed. To evaluate this possibility, we implemented a procedural change in Experiment 2: On all days, the rats were allowed daily access to water for an additional $10 \mathrm{~min}, 2 \mathrm{~h}$ following the experimental session.

A second contributing factor to the less-than-optimal suppression observed in Experiment 1 might involve the use of the single-bottle testing procedure. Because Dragoin, McCleary, and McCleary (1971) and Grote and Brown (1971) have shown that the single-bottle assessment is not a particularly reliable measure of aversion, the more sensitive two-bottle preference test was employed in Experiment 2.

A third change in this experiment was that the experiment also assessed the effect(s) of shifting the testing procedure (i.e., single-bottle animals were shifted to the two-bottle procedure, and vice versa).

\section{Method}

Subjects and Apparatus. Twenty male Holtzman rats served as subjects. The animals were housed and maintained as in Experiment 1. All fluid-consumption tests were conducted in the home cage.

Procedure. Deprivation and baseline procedures were the same as those employed in Experiment 1, with one exception: all subjects received an additional $10 \mathrm{~min}$ of access to water $2 \mathrm{~h}$ following the daily experimental session.

Four equal-sized groups $(n=5)$ were formed at the start of the 5day baseline period. Two of these groups were randomly designated to receive the 1 part denatonium saccharide per 1,000 parts water solution during Phases 1 and 2; the remaining two groups were designated to receive the 1 part per 5,000 solution during Phases 1 and 2 . One group receiving each concentration received single-bottle testing during Phase 1; the second group received two-bottle testing. Phase 1 lasted 15 days. Those subjects tested under the single-bottle condition received five 3-day fluid-presentation cycles identical to those administered in Experiment 1. The two-bottle preference test involved the simultaneous presentation of separate centrifuge tubes, one containing water and one containing a denatonium solution. The placement of centrifuge tubes on the home cage for the two-bottle test was randomized daily for each subject.

The Phase 1 testing procedures were reversed for Phase 2 (6 days): animals that had received the single-bottle procedure during Phase 1 received the two-bottle procedure during Phase 2 , and vice versa. The denatonium concentration received by the specific groups remained unchanged from Phase 1 to Phase 2.

\section{Results}

Group mean consumption scores for baseline and Phase 1 for the single-bottle test are shown in Figure 2. Group means for these two phases for the two-bottle test are shown in Figure 3. An analysis of the baseline data compared all four groups. As this analysis failed to yield significance for the groups factor $[F(3,16)=.16]$, the assumption of equality at the start of testing was accepted.

Phase 1. Separate analyses compared the $1 / 1,000$ and $1 / 5,000$ groups under the single-bottle and two-bottle conditions, respectively, for Phase 1 . During Phase 1 the days factor was found to be significant $[F(14,112)=$ $23.90, p<.001]$ for the single-bottle condition (see Figure 2). Subsequent Newman-Keuls tests indicated that 


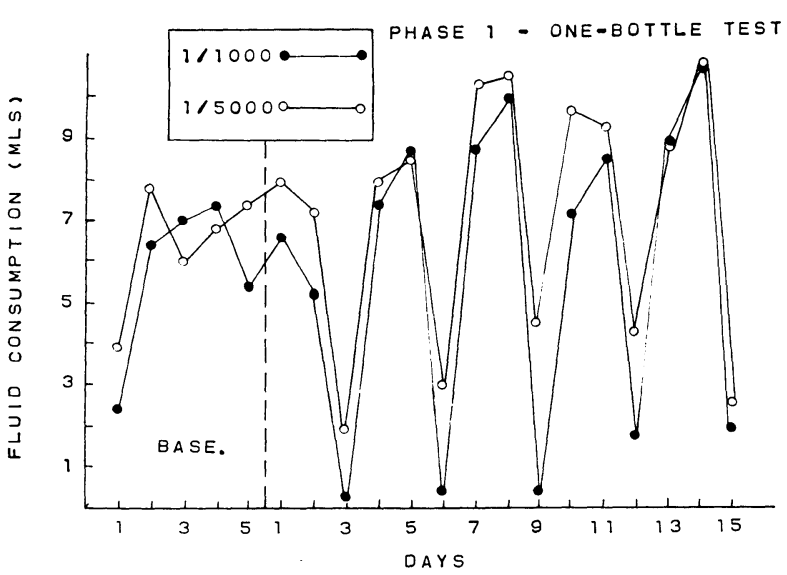

Figure 2. Mean fluid consumption scores (in milliliters) for the last 2 days of baseline and Phase 1 (single-bottle test), Experiment 2. Denatonium saccharide was presented on Days 3, 6, 9, 12, and 15 of Phase 1.

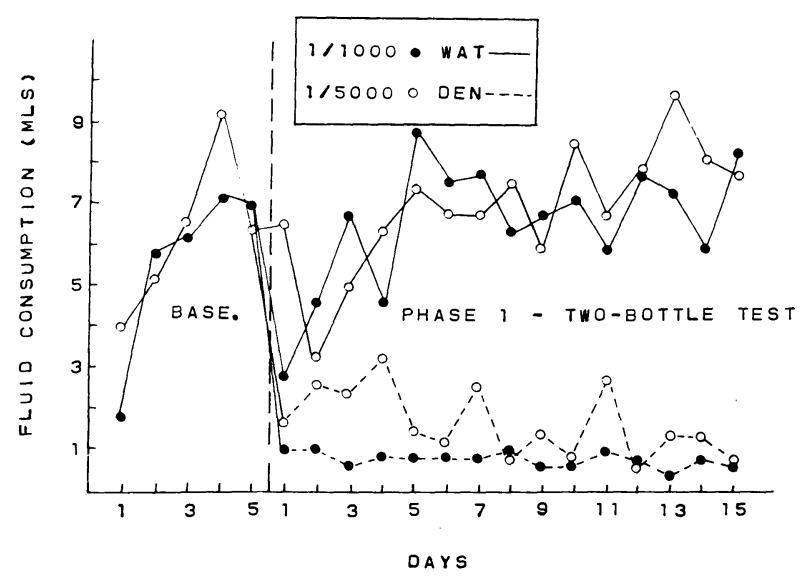

Figure 3. Mean fluid consumption scores (in milliliters) for the last 2 days of baseline and Phase 1 (two-bottle test), Experiment 2.

both groups consumed significantly $(p<.01)$ less fluid on days when denatonium saccharide was presented (Days $3,6,9,12$, and 15). Analysis of the two-bottle eonsumption scores yielded significance for the type of fluid $[F(1,8)$ $=178.07, p<.001]$, days $[F(14,112)=2.19, p=$ $.012]$, and type of fluid $\times$ days $[F(14,112)=3.38, p<$ $.001]$ effects. Clearly, both the $1 / 1,000$ and $1 / 5,000$ animals displayed an immediate and lasting preference for water over denatonium saccharide (see Figure 3). Given that significantly $(p<.05)$ more of the $1 / 5,000$ than the $1 / 1,000$ solution of denatonium saccharide was consumed on Days 2, 3, 4, 7, and 11, it would appear that the $1 / 1,000$ solution had the greatest suppressive effect. This is in agreement with the results of Experiment 1.

Phase 2. As can be seen from Figure 4, the reversal in testing conditions resulted in an immediate adaptation to the new fluid-consumption regimen of Phase 2. Analysis of the single-bottle consumption scores showed significance for the days factor $[F(5,40)=56.42, p<.001]$. Newman-Keuls tests indicated that both groups consumed significantly $(p<.01)$ less fluid on Days 3 and 6 when denatonium saccharide was presented. Analysis of the two-bottle scores yielded significance for the type of fluid $[F(1,8)=293.54, p<.001]$ and days $\times$ type of fluid $[F(5,40)=3.57, p=.009]$ effects. As in Phase 1, the two-bottle procedure resulted in an immediate preference for water over either the $1 / 1,000$ or the $1 / 5,000$ solution of denatonium saccharide. It was of interest to note that Group 1/5,000 tended to consume more of the denatonium solution than did Group 1/1,000, but this difference achieved statistical reliability $(p<.05)$ only on Day 6 .

\section{GENERAL DISCUSSION}

When denatonium saccharide was the only fluid available, the animals adapted to, and consumed, strong concentrations of this bitter compound (e.g., 1/1,000 and 1/5,000). However, the suppressive effects of the $1 / 1,000$ and $1 / 5,000$ solutions were more pronounced and long lasting when the animals were presented with a two-bottle choice between water and these denatonium saccharide solutions than when they had no choice (see Figure 2). These strong aversions indicate that denatonium saccharide, especially in the $1 / 1,000$ concentration, has the potential to act as a deterrent.

The results for Groups 10 and 100 in Experiment 1 suggest that denatonium saccharide in more diluted concentrations $(1 / 10,000$ and $1 / 100,000)$ is not sufficiently aversive to suppress consumption. One might be tempted to propose that these animals were not capable of discriminating between these solutions and plain tap water. This speculation, however, awaits further experimental verification, given the results of the two-bottle choice test of Experiment 2.

Shifting the testing procedure (Phase 2, Experiment 2) failed to result in any noticeable change in the suppressive effects of either concentration of denatonium saccharide. This finding supports the view that the subjects were reacting to the physical properties of the bitter substance, rather than to some specific property of the testing schedule (e.g., the cyclic schedule employed under the single-bottle procedure).

In summary, denatonium saccharide at certain concentrations is sufficiently aversive to alter fluid consumption in at least this species of rodent. The question is whether the pairing of this bitter substance with a second taste (e.g., saccharin) will result in suppressed intake of the second taste when it is subsequently presented alone. Positive results would suggest new and potentially fruitful applications for denatonium

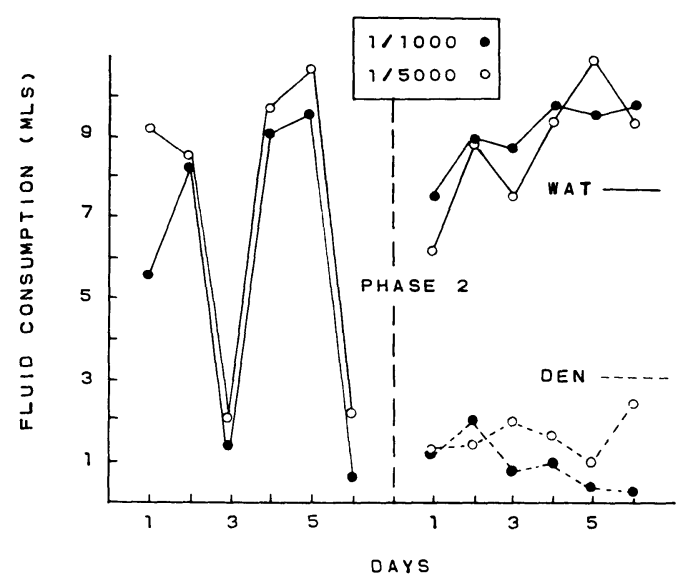

Figure 4. Mean fluid consumption scores in (milliliters) following the shift in testing conditions (i.e., Phase 2), Experiment 2. Single-bottle test results are shown in the left panel with denatonium saccharide being presented on Days 3 and 6. Two-bottle test results are shown in the right panel. 
saccharide as a deterrent to crop ingestion. If such pairings prove to be ineffective, then a determination of the ability of denatonium saccharide to become associated with toxicosis in the more traditional conditioned taste-aversion paradigm would appear to be in order. Finally, additional testing employing a variety of species is needed to determine the generalizability of denatonium saccharide's aversiveness.

\section{REFERENCES}

Berning, C. K., Griffith, J. F., \& Wild, J. E. (1982). Research on the effectiveness of denatonium benzoate as a deterrent to liquid de- tergent ingestion by children. Fundamentals of Applied Toxicology, 2, 44-48.

Dragoin, W., McCleary, G. E., \& McCleary, P. (1971). A comparison of two methods of measuring conditioned taste aversions. Behavior Research Methods \& Instrumentation, 3, 309-310.

Grote, F. W., \& Brown, R. T. (1971). Conditioned taste aversions: Two-stimulus tests are more sensitive than one-stimulus tests. Behavior Research Methods \& Instrumentation, 3, 311-312.

(Manuscript received for publication February 6, 1986.) 